\title{
Zero-Knowledge Proofs and String Commitments Withstanding Quantum Attacks
}

\author{
Ivan Damgård ${ }^{1}$, Serge Fehr ${ }^{2, \star}$, and Louis Salvail ${ }^{1}$ \\ 1 BRICS, FICS, Aarhus University, Denmark ${ }^{\star \star}$ \\ $\{$ ivan, salvail\}@brics.dk \\ 2 CWI, Amsterdam, The Netherlands \\ fehr@cwi.nl
}

\begin{abstract}
The concept of zero-knowledge (ZK) has become of fundamental importance in cryptography. However, in a setting where entities are modeled by quantum computers, classical arguments for proving ZK fail to hold since, in the quantum setting, the concept of rewinding is not generally applicable. Moreover, known classical techniques that avoid rewinding have various shortcomings in the quantum setting.

We propose new techniques for building quantum zero-knowledge (QZK) protocols, which remain secure even under (active) quantum attacks. We obtain computational QZK proofs and perfect QZK arguments for any NP language in the common reference string model. This is based on a general method converting an important class of classical honestverifier ZK (HVZK) proofs into QZK proofs. This leads to quite practical protocols if the underlying HVZK proof is efficient. These are the first proof protocols enjoying these properties, in particular the first to achieve perfect QZK.

As part of our construction, we propose a general framework for building unconditionally hiding (trapdoor) string commitment schemes, secure against quantum attacks, as well as concrete instantiations based on specific (believed to be) hard problems. This is of independent interest, as these are the first unconditionally hiding string commitment schemes withstanding quantum attacks.

Finally, we give a partial answer to the question whether QZK is possible in the plain model. We propose a new notion of QZK, non-oblivious verifier QZK, which is strictly stronger than honest-verifier QZK but weaker than full QZK, and we show that this notion can be achieved by means of efficient (quantum) protocols.
\end{abstract}

\section{Introduction}

Since its introduction by Goldwasser, Micali and Rackoff [14], the concept of zero-knowledge $(\mathrm{ZK})$ proof has become a fundamental tool in cryptography. In-

\footnotetext{
* Research was carried out while at the Centre for Advanced Computing - Algorithms and Cryptography, Department of Computing, Macquarie University, Australia.

** BRICS stands for Basic Research in Computer Science (www.brics.dk) and FICS for Foundations in Cryptography and Security, both funded by the Danish Natural Sciences Research Council.
} 
formally, in a ZK proof of a statement, the verifier learns nothing beyond the validity of the statement. In particular, everything the verifier can do as a result of the interaction with the prover during the ZK proof, the verifier could also do "from scratch", i.e., without interacting with the prover. This is argued by the existence of an efficient simulator which produces a simulated transcript of the execution, indistinguishable from a real transcript. ZK protocols exist for any NP language if one-way functions exist [2,3,15], also more efficient solutions are known for specific languages like Quadratic-Residuosity [14] or GraphIsomorphism [15].

From a theoretical point of view, it is natural to ask whether such classical protocols are still secure if cheating players are allowed to run (polynomial time bounded) quantum computers. But the question also has some practical relevance: although quantum computers may not be available to the general public in any foreseeable future, even a single large scale quantum computer could be used to attack the security of existing protocols.

To study this question, two issues are important. First, the computational assumption on which the protocol is based must remain true even if the adversary is quantum. This rules out many assumptions such as hardness of factoring or extracting discrete logs [23], but a few candidates still remain, for instance some problems related to lattices or error correcting codes. In general, it is widely believed that quantum one-way functions exist, i.e., functions that are easy to compute classically, but hard to invert, even on a quantum computer.

A second and more difficult question is whether the proof of security remains valid against a quantum adversary. A major problem in this context comes from the fact that in the classical definition of ZK, the simulator is allowed to rewind the verifier in order to generate a simulated transcript of the protocol execution. However, if prover and verifier are allowed to run quantum computers, rewinding is not generally applicable, as it was originally pointed out by Van de Graaf [27]. We discuss this in more detail later, but intuitively, the reason is that when a quantum computer must produce a classical output, such as a message to be sent, a (partial) measurement on its state must be done. This causes an irreversible collapse of the state, so that it is not generally possible to reconstruct the original state. Moreover, copying the verifier's state before the measurement is forbidden by the no-cloning theorem. Therefore, protocols that are proven ZK in the classical sense using rewinding of the verifier may not be secure with respect to a quantum verifier. This severe breakdown of the classical concept of ZK in a quantum world is the motivation of this work.

It is well known that rewinding can cause "problems" already in a classical setting. In particular, it has been realized that rewinding the verifier limits the composability of ZK protocols. As a result, techniques have been proposed that avoid rewinding the verifier, for instance the non-black-box ZK technique from [1], or - in the common reference string model - techniques providing concurrent ZK [13,22,9], non-interactive ZK [4] or universally-composable (UC) ZK $[5,6,11]$ and related models [21]. One might hope that some of these ideas would translate easily to the quantum setting. 
However, the non-black box technique from [1] is based on the simulator using the verifier's program and current state to predict its reaction to a given message. Doing so for a quantum verifier will collapse its state when a measurement is done to determine its next message, so it is not clear that this technique will generalize to a quantum setting. The known constructions of UCZK protocols and non-interactive ZK are all based on computational assumptions that are either false in a quantum setting or for which we have no good candidate for concrete instantiations: the most general sufficient assumption is the existence of one-way trapdoor permutations (i.e. as far as we know) but all known candidates are easy to invert on a quantum computer. Regardless of this type of problem, great care has to be taken with the security proof: despite the fact that the simulator in the UC model must not use rewinding, it is not true that a security proof in the UC model automatically implies security against quantum adversaries - we discuss this in more details later in the paper. Finally, the technique for concurrent ZK from [9] avoids rewinding the verifier but instead rewinds the prover to prove soundness, leading to similar problems.

Before describing our results, we note that quantum zero-knowledge proof systems were already studied from a complexity theoretic point of view by Watrous in [26]. The proof systems considered there all assume the prover to be computationally unbounded and the zero-knowledge condition is only enforced against honest verifiers. Clearly, these restrictions make those proof systems unsuitable for cryptographic applications. In this paper, we focus on efficient quantum zero-knowledge protocols in a cryptographic setting.

We propose three distinct techniques applicable to an important class of (classical) honest-verifier ZK (HVZK) proofs (in which the verifier is guaranteed to follow the protocol), namely so-called $\Sigma$-protocols (3-move public-coin protocols). We convert such protocols into quantum zero-knowledge (QZK) proofs, which are ZK (as well as sound) even with respect to (active) quantum attacks. In all cases, the new proof protocol proceeds in three moves like the underlying $\Sigma$-protocol, and its overhead in terms of communication is reasonable. To the best of our knowledge, these are the first (practical) zero-knowledge proofs withstanding active quantum attacks.

The first technique assumes the existence of an unconditionally hiding trapdoor string commitment scheme (secure against quantum attacks) and can be proven secure in the common-reference-string (CRS) model. It requires only classical computation and communication and achieves perfect or statistical QZK, assuming the underlying $\Sigma$-protocol was perfect or statistical HVZK, and is an interactive argument (computationally sound). The communication overhead of the new QZK protocol in comparison with the underlying $\Sigma$-protocol is essentially given by communicating and opening one string commitment. The technique directly implies perfect or statistical QZK arguments for NP.

This first approach requires addressing the problem of constructing unconditionally hiding and computationally binding trapdoor string commitment schemes withstanding quantum attacks. This is non-trivial since the classical definition of computational binding cannot be used for a quantum adversary as 
it was pointed out in [12] with respect to bit commitments and in [8] with respect to string commitments. In fact, it was not even clear how computational binding for a string commitment should be defined. In [8], a computational binding condition was introduced with their application in mind but no concrete instance was proposed.

We propose a new definition of computational binding that is strong enough for our (and other) applications. On the other hand, we propose a generic construction for schemes satisfying our definition based on special-sound $\Sigma$ protocols for hard-to-decide languages, and we give examples based on concrete intractability assumptions. Our construction yields the first unconditionally hiding string commitment schemes withstanding quantum attacks, under concrete as well as under general intractability assumptions. Moreover, since our definition implies the one from [8], our schemes can be used to provide secure quantum oblivious transfer.

The second technique assumes the existence of any quantum one-way function and is also secure in the CRS model. It requires classical communication and computation and produces computational QZK interactive proofs for any NP language. It can be efficiently instantiated under more specific complexity assumptions.

The last technique requires no computational assumption and is provably secure in the plain model (no CRS). However, it requires quantum computation and communication and does not achieve full QZK but what we call non-oblivious verifier $Q Z K$. This new notion is weaker than QZK but strictly stronger than honest-verifier QZK (as defined in [26]). Essentially, a non-oblivious verifier may arbitrarily deviate from the protocol but still generates all private and public classical random variables available to the honest verifier according the same distribution. The (quantum) communication complexity of the non-oblivious verifier QZK proof essentially equals the (classical) communication complexity of the underlying $\Sigma$-protocol.

The paper is organized as follows. In Sect. 2, we introduce some relevant notations. We also argue why rewinding causes a problem in a quantum setting and why UCZK does not imply QZK. In Sect. 3, we define and construct the unconditionally hiding (trapdoor) commitment schemes used in Sect. 4 for QZK proofs in the common-reference-string model. Finally, the non-oblivious verifier QZK proof in the plain model is presented in Sect. 5.

Due to space limitations, some descriptions and discussions appear in a shortened form in this proceedings version, they appear in full in the full version [10].

\section{Preliminaries}

\subsection{Zero-Knowledge Interactive Proofs}

The Classical Case: We assume the reader to be familiar with the classical notions of (HV)ZK interactive proofs (and arguments) and of (special-sound) $\Sigma$-protocols. We merely fix some notation and terminology here. For an introduction to these concepts we refer to the full version of this paper [10] or to the literature. 
Let $R=\{(x, w)\}$ be a binary relation. Write $L_{R}=\{x \mid \exists w:(x, w) \in R\}$ for the language defined by $R$. For $x \in L_{R}$, any $w$ such that $(x, w) \in R$ is called a witness (for $x \in L$ ), and we write $W_{R}(x)=\{w \mid(x, w) \in R\}$ for the set of witnesses for $x \in L$. We assume that the size of the witnesses for $x \in L$ are polynomially bounded by the size of $x$, and that $R$ is poly-time testable.

We refer to a $\Sigma$-protocol (P, V) for a language $L$ by a triple (a, c, z), where we understand $\mathrm{a}, \mathrm{c}$ and $\mathrm{z}$ as the processes of choosing/computing the first message $a$, the (random) challenge $c$ and the corresponding answer $z$, respectively, as specified by the protocol (with some input $x \in L$ ), and we write $a \leftarrow \mathrm{a}, c \leftarrow \mathrm{c}$ and $z \leftarrow z_{x}(a, c)$, respectively, for the execution of these processes. Furthermore,

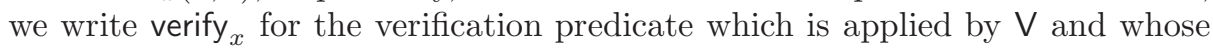
output accept or reject, respectively 0 or 1 , determines whether $V$ should accept the proof or not. We stress that when considering a computationally bounded (honest) prover $\mathrm{P}$ as we do here the answer $z$ is typically not computed by $\mathrm{P}$ as a function of $a, c$ and $x$ (as the notation $z \leftarrow \mathrm{z}_{x}(a, c)$ might suggest), but rather as a function of the randomness used to generate $a$, of the challenge $c$ and of a witness $w \in W_{R}(x)$. Per default, we understand a $\Sigma$-protocol to be unconditionally sound. Clearly, for a fixed $x \notin L$, the soundness error $\epsilon$ of such a $\Sigma$-protocol is given by the maximum over all possible first messages $a$ of the fraction of the possible challenges $c$ for $a$ that allow an answer $z$ which is accepted by $\mathrm{V}$.

It is known that statistical ZK $\Sigma$-protocols only exist for languages $L \in$ co-AM. Most of the well-known $\Sigma$-protocols are proof-system for languages that are trivial on a quantum computers. However, some languages like graph isomorphism (i.e. GI) have special sound $\Sigma$-protocols and are not known to be trivial on a quantum computer. This is also the case for some recently proposed lattice problems [19]. It is not known whether co-AM can be efficiently recognized by a quantum computer.

The Quantum Case: ZK quantum interactive proof systems are defined as the natural generalization of their classical counterpart and were introduced and first studied by Watrous [24,26]. Quantum ZK (QZK) is defined as for the classical case except that the quantum simulator is required to produce a state that is exponentially close, in the trace-norm sense, to the verifier's view. Formal definitions for QZK proof systems can be found in the full version [10].

\subsection{The Problem with Quantum Rewinding}

Rewinding a party to a previous state is a common proof technique for showing the security of many different kinds of protocols in the computational model. In general, this technique cannot be applied when the party is modeled by a quantum computer. Originally observed by Van de Graaf [27], this implies that security proofs of many well-established classical protocols do not hold if one party is running a quantum computer even if the underlying assumption under which the security proof holds withstands quantum attacks.

Rewinding is in general not possible since taking a snapshot of a quantum memory is tantamount to quantum cloning. Unlike in the classical case, there 
is no way to copy a quantum memory regardless of what the memory contains. The only generic way to restore a quantum memory requires to re-generate it from scratch. Proceeding that way may not be possible efficiently.

One consequence of the no quantum rewinding paradigm is particularly relevant to us. Sequential repetitions of an HVZK $\Sigma$-protocol for a language $L$ results in a ZK protocol for $L$ with negligible soundness error. It follows that this straightforward construction is not guaranteed to be secure against quantum verifiers.

Another example is the use of rewinding for proving secure applications of computationally binding commitment schemes. Such a security proof is done by showing that an attacker that breaks the application can be used to compute two different openings of a commitment and thus to break the binding property of the commitment scheme. This reduction, however, requires typically to rewind of the attacker, and thus by the no quantum rewinding paradigm does not yield a valid security proof in a quantum setting.

More details can be found in the full version [10].

\subsection{UCZK Does Not Imply QZK}

In [5], Canetti proposes a new framework for defining and proving cryptographic protocols secure: the universal composability (UC) framework. This framework allows to define and prove secure cryptographic protocols as stand-alone protocols, while at the same time guaranteeing security in any application by means of a general composition theorem. The UC security definition essentially requires that the view of any adversary attacking the protocol can be simulated while in fact running an idealized version of the protocol, which essentially consists of a trusted party called ideal functionality. The simulation should be indistinguishable for any distinguisher, called environment, which may be on-line, and provides the inputs and receives the outputs. Furthermore, the UC definition explicitly prohibits rewinding of the environment and thus of the adversary (as it may communicate with the environment). This restriction is crucial for the proof of the composition theorem. We refer to [5] for more details.

Since the UC framework forbids rewinding the adversary, it seems that UCZK implies QZK, assuming the underlying computational assumption withstands quantum attacks. This intuition is false in general. The reason being that even though the UC framework does not allow the simulator to rewind the adversary, it is still allowed to use rewinding as a proof-technique in order to show that the simulator produces a "good" simulation. For instance, it is allowed to argue that if an environment can distinguish the simulation from a real protocol execution, then by rewinding the environment together with the adversary one can solve efficiently a problem assumed to be hard. We illustrate this on a concrete example in $[10]$.

\section{Unconditionally Hiding (Trapdoor) Commitments}

In this section we study and construct classical (trapdoor) commitment schemes secure against quantum attacks. In contrast to quantum commitment schemes, 
such schemes do not require quantum computation (in order to compute, open or verify commitments), but they are guaranteed to remain secure even under quantum attacks. Our construction, which is based on hard-to-decide languages with special-sound $\Sigma$-protocols, yields the first unconditionally hiding string commitment schemes withstanding quantum attacks. In Sect. 4, we use these commitments to construct QZK proofs. A further application of our commitment schemes is given in [10], where it is shown how they give rise to quantumly secure oblivious transfer.

\subsection{Defining Security in a Quantum Setting}

Informally, by publishing a commitment $C=\operatorname{commit}_{p k}(s, \rho)$ for a random $\rho$, a commitment scheme allows a party to commit to a secret $s$, such that the commitment $C$ reveals nothing about the secret $s$ (hiding property) while on the other hand the committed party can open $C$ to $s$ by publishing $(s, \rho)$ but only to $s$ (binding property).

Formally, a commitment scheme (of the kind we consider) consists of two poly-time algorithms: A key-generation algorithm $\mathcal{G}$ which takes as input the security parameter $\ell$ and specifies an instance of the scheme by generating a public$k e y p k$, and an algorithm commit which allows to compute $C=\operatorname{commit}_{p k}(s, \rho)$ given a public-key $p k$ as well as $s$ and $\rho$ chosen from appropriate finite sets $\mathcal{S}$ and $\mathcal{R}$ (specified by $p k$ ). $\mathcal{S}$ is called the domain of the commitment scheme. Classically, the hiding property is formalized by the non-existence of a distinguisher which is able to distinguish $C=\operatorname{commit}_{p k}(s, \rho)$ from $C=\operatorname{commit}_{p k}\left(s^{\prime}, \rho^{\prime}\right)$ with non-negligible advantage, where $s, s^{\prime} \in \mathcal{S}$ are chosen by the distinguisher and $\rho, \rho^{\prime} \in \mathcal{R}$ are random. On the other hand, the binding property is formalized by the non-existence of a forger able to compute $s, s^{\prime} \in \mathcal{S}$ and $\rho, \rho^{\prime} \in \mathcal{R}$ such that $s \neq s^{\prime}$ but commit $_{p k}(s, \rho)=\operatorname{commit}_{p k}\left(s, \rho^{\prime}\right)$. If the distinguisher respectively the forger is restricted to be poly-time, then the scheme is said to be computationally hiding respectively binding, while without restriction on the distinguisher respectively the forger, it is said to be unconditionally hiding respectively binding.

In order to define security of such a commitment scheme (G, commit) in a quantum setting, the (computational or unconditional) hiding property can be adapted in a straightforward manner by allowing the distinguisher to be quantum. The same holds for the unconditional binding property, which is equivalent to requiring that every $C$ uniquely defines $s$ such that $C=\operatorname{commit}_{p k}(s, \rho)$ for some $\rho$. However, adapting the computational binding property in a similar manner simply by allowing the forger to be quantum results in a too weak definition. The reason being that in order to prove secure an application of a commitment scheme, which is done by showing that an attacker that breaks the application can be transformed in a black-box manner into a forger that violates the binding property, the attacker typically needs to be rewound, which cannot be justified in a quantum setting by the no-quantum-rewinding paradigm as discussed in Sect. 2.2. The following definition for the computational binding property of a commitment scheme with respect to quantum attacks is strong enough to prove 
secure applications (as in Sect. 4 and in [10]) based on the security of the underlying commitment scheme, but it is still weak enough in order to prove the binding property for concrete commitment schemes (see Sect. 3.2 and 3.3).

Let $(\mathcal{G}$, commit) be a commitment scheme as introduced above, and let $\mathcal{S}$ denote its domain. Informally, we require that it is infeasible to produce a list of commitments and then open (a subset of) them in a certain specified way with a probability significantly greater than expected. We formalize this as follows. Let $Q$ be a predicate of the following form. $Q$ takes three inputs: (1) a non-empty set $A \subseteq\{1, \ldots, N\}$ where $N$ is upper bounded by a polynomial in $\ell$, (2) a tuple $s_{A}=\left(s_{i}\right)_{i \in A}$ with $s_{i} \in \mathcal{S}$, and (3) an element $u \in \mathcal{U}$ where $\mathcal{U}$ is some finite set; and it outputs $Q\left(A, s_{A}, u\right) \in\{0,1\}$. We do not require $Q$ to be efficiently computable. Consider a polynomially bounded quantum forger $\mathcal{F}$ in the following game: $\mathcal{F}$ takes as input $p k$, generated by $\mathcal{G}$, and announces commitments $C_{1}, \ldots, C_{N}$. Then, it is given a random $u \in \mathcal{U}$, and it outputs $A, \boldsymbol{s}_{A}=\left(s_{i}\right)_{i \in A}$ and $\boldsymbol{\rho}_{A}=\left(\rho_{i}\right)_{i \in A} \cdot \mathcal{F}$ is said to win the game if $Q\left(A, s_{A}, u\right)=1$ and $C_{i}=\operatorname{commit}_{p k}\left(s_{i}, \rho_{i}\right)$ for every $i \in A$. We require that every forger has essentially the same success probability in winning the game as when using an ideal (meaning unconditionally binding) commitment scheme (where every $C_{i}$ uniquely defines $s_{i}$ ). In the latter case, the success probability is obviously given by $p_{\mathrm{IDEAL}}=\max _{\boldsymbol{s} \in \mathcal{S}^{N}}\left|s a t_{Q}(\boldsymbol{s})\right| /|\mathcal{U}|$ with $\operatorname{sat}_{Q}(s)=\left\{u \in \mathcal{U} \mid \exists A: Q\left(A, s_{A}, u\right)=1\right\}$, where $s_{A}$ stands for the restriction of $s$ to its coordinates $s_{i}$ with $i \in A$. In this definition, $Q$ models a condition that must be satisfied by the opened value in order for the opening to be useful for the committer. For each application scenario, such a predicate can be defined.

Definition 1. A commitment scheme (G, commit) is called computational Qbinding if for every predicate $Q$, every polynomially bounded quantum forger $\mathcal{F}$ wins the above game with probability $p_{\mathrm{REAL}}=p_{\mathrm{IDEAL}}+a d v$, where adv, the advantage of $\mathcal{F}$, is (negative or) negligible (in $\ell$ ).

It is not hard to verify that in a classical setting (where $\mathcal{F}$ is allowed to be rewound), the classical computational binding property is equivalent to the above computational Q-binding property. Furthermore, it is rather obvious that the computational Q-binding property for a commitment scheme with domain $\mathcal{S}$ implies the computational Q-binding property for the natural extension of the scheme to the domain $\mathcal{S}^{k}$ (for any $k$ ) by committing componentwise. Note that this desirable preservation of the binding property does not hold for the binding property introduced in [8].

Finally, we define a trapdoor commitment scheme ${ }^{1}$ as a commitment scheme in the above sense with the following additional property. Besides the publickey $p k$, the generator $\mathcal{G}$ also outputs a trapdoor $\tau$ which allows to break either the hiding or the binding property. Specifically, if the scheme is unconditionally binding, then $\tau$ allows to efficiently compute $s$ from $C=\operatorname{commit}_{p k}(s, \rho)$, and if it is unconditionally hiding, then $\tau$ allows to efficiently compute commitments $C$ and correctly open them to any $s$.

\footnotetext{
${ }^{1}$ Depending on its flavor, a trapdoor commitment scheme is also known as an extractable respectively as an equivocable or a chameleon commitment scheme.
} 


\subsection{A General Framework}

In this section, we propose a general framework for constructing unconditionally hiding and computationally Q-binding (trapdoor) string commitment schemes. For that, consider a language $L=L_{R}$ and assume that

1. $L$ admits a (statistical) HVZK special-sound $\Sigma$-protocol $\Pi=(\mathrm{a}, \mathrm{c}, \mathrm{z})^{2}$,

2. there exists an efficient generator $\mathcal{G}_{\text {yes }}$ generating $x \in L$ together with a witness $w \in W_{R}(x)$ (more precisely, $\mathcal{G}_{\text {yes }}$ takes as input security parameter $\ell$ and outputs $x \in L$ of bit size $\ell$ and $w \in W_{R}(x)$ ), and

3. for all poly-size quantum circuits $\mathcal{D}$ and polynomials $p(\ell)>0$, if $\ell$ is large enough then there exists $x_{\text {no }} \notin L$ of bit size $\ell$ such that for $x_{\text {yes }}$ generated by $\mathcal{G}_{\text {yes }}$ (on input $\ell$ )

$$
\mid \operatorname{Pr}\left(\mathcal{D}\left(x_{\text {yes }}\right)=\text { yes }\right)-\operatorname{Pr}\left(\mathcal{D}\left(x_{\text {no }}\right)=\text { yes }\right) \mid<1 / p(\ell) .
$$

Note that 3 . only requires that for every distinguisher $\mathcal{D}$ it is hard to distinguish a randomly generated yes-instance $x \in L$ from some no-instance $x \notin L$, which in particular may depend on $\mathcal{D}$.

Given such $L$, the construction in Fig. 1 provides an unconditionally hiding trapdoor commitment scheme. We assume that c samples challenge $c$ randomly from $\{0,1\}^{t}$ for some $t$.

$\mathcal{G}$ is given by $\mathcal{G}_{\text {yes }}$, where the generated $x \in L$ is parsed as public key $p k$ and $w \in W_{R}(x)$ as trapdoor $\tau$. The domain $\mathcal{S}$ is defined to be $\mathcal{S}=\{0,1\}^{t}$.

commit $_{p k}$ : To commit to $s \in \mathcal{S}=\{0,1\}^{t}$, use the HVZK simulator for $\Pi$ to generate $(a, c, z)$. Set $C=(a, s \oplus c)$ to be the commitment for $s$.

A commitment $C=(a, d)$ is opened to $s$ by announcing the corresponding values $c$ and $z$, and such an opening is accepted if and only if $s \oplus c=d$ and verify $_{x}(a, c, z)=$ accept.

Fig. 1. Trapdoor commitment scheme (G, commit).

If $\Pi$ is special HVZK, meaning that $(a, c, z)$ can be simulated for a given $c$, then the commitment scheme can be slightly simplified: $(a, c, z)$ is generated such that $c=s$ and $C$ is simply set to be $C=a$.

Theorem 1. Under assumption 3., (G, commit) in Fig. 1 is an unconditionally hiding and computationally Q-binding trapdoor commitment scheme.

\footnotetext{
${ }^{2}$ As will become clear, the prover's efficiency in the $\Sigma$-protocol does not influence the efficiency of the resulting commitment scheme as far as the committer and the receiver are concerned. An efficient prover is only required if one wants to take advantage of the trapdoor.
} 
As will become clear from the proof below, if the underlying $\Sigma$-protocol $\Pi$ is perfect HVZK, then (G, commit) is perfectly binding in the sense that there exists no distinguisher with non-zero advantage, meaning that a commitment $C$ for $s$ is statistically independent of $s$.

Proof. It is clear that a correct opening is accepted. It is also rather obvious that the scheme is unconditionally hiding: The distribution of $(a, c, z)$ generated by the HVZK simulator is statistically close to the distribution of $(a, c, z)$ generated by the protocol. There, however, $c$ is chosen independently of $a$. Therefore, $a$ gives essentially no information on $c$ and thus $C=(a, s \oplus c)$ gives essentially no information on $s$ (as $s \oplus c$ acts as a one-time pad). The trapdoor property can be seen as follows. Knowing the trapdoor $\tau=w$, put $C=(a, d)$ where $a \leftarrow$ a and $d$ is randomly sampled from $\{0,1\}^{t}$. Given arbitrary $s \in\{0,1\}^{t}$, compute $c=d \oplus s$ and $z \leftarrow \mathbf{z}_{x}(a, c)$ using the witness $w$ (and the randomness for the generation of $a)$. It is obvious that $(s, c, z)$ opens $C$ correctly to $s$.

It remains to show the computational Q-binding property. We show that if there exists a forger $\mathcal{F}$ that can break the Q-binding property of the commitment scheme (without knowing the trapdoor) for some predicate $Q$ according to Definition 1 , then there exists a circuit $\mathcal{D}$ that contradicts assumption $3 . \mathcal{D}$ is illustrated in Figure 2 and is quantum if and only if $\mathcal{F}$ is.

$\mathcal{D}$ : The input is $x$, either in $L$ or not in $L$.

1. Invoke $\mathcal{F}$ with public-key $p k=x$ in order to get commitments $C_{1}, \ldots, C_{N}$,

2. Pick random $u \in \mathcal{U}$ and announce it to $\mathcal{F}$,

3. $\mathcal{F}$ announces $A \subseteq\{1, \ldots, N\}$ and, for $i \in A$, tries to open $C_{i}$ to $s_{i}$ such that $Q\left(A, s_{A}, u\right)=1$ for $s_{A}=\left(s_{i}\right)_{i \in A}$,

4. Verify the openings and whether indeed $Q\left(A, s_{A}, u\right)=1$, if successful then output yes and otherwise no.

Fig. 2. Distinguisher $\mathcal{D}$ for $x \in L$ versus $x \notin L$.

If $x$ is generated by $\mathcal{G}_{\text {yes }}$ then $p k=x$ is a valid public-key for the commitment scheme with the right distribution and thus $\operatorname{Pr}(\mathcal{D}(x)=$ yes $)=p_{\text {REAL }}=p_{\text {IDEAL }}+a d v$ where $a d v$ is $\mathcal{F}$ 's advantage. On the other hand, if $x \notin L$, then by the special soundness property of $\Pi$, given $a$ there is only one $c$ that allows an answer $z$ such that verify ${ }_{x}(a, c, z)=$ accept. Hence, for any $C_{i}$ there is only one $s_{i} \in \mathcal{S}$ to which $C_{i}$ can be successfully opened. Therefore, $\operatorname{Pr}(\mathcal{D}(x)=$ yes $) \leq p_{\text {IDEAL }}$. If $a d v$ is (positive and) non-negligible, then this contradicts 3.

We would like to point out once more that our definition of the (computational) binding property inherits the following feature. If a commitment scheme with domain $\mathcal{S}$ is computational Q-binding, then its natural extension to a commitment scheme with domain $\mathcal{S}^{k}$ by committing componentwise (with the 
same $p k$ ) is also computational Q-binding. In particular, any computational Qbinding bit commitment scheme gives rise to a computational Q-binding string commitment scheme.

\subsection{Concrete Instantiations}

We propose three concrete languages which are believed to be hard to decide as required in the above section and which admit HVZK special-sound $\Sigma$-protocols. The first language is based on a problem from coding theory: the Code-Equivalence (CE) problem. It requires to decide whether two generator matrices generate the same code up to a permutation of the coordinates, and it is known to be at least as hard (in the worst case) as the Graph-Isomorphism (GI) problem. Furthermore, it admits a similar $\Sigma$-protocol as GI. Finally, and in contrast to GI, there is a generator believed to produce hard yes-instances. More details are given in [10].

The next two languages are gap versions of the famous lattice problems Shortest-Vector and Closest-Vector, where the no-instances are promised to be "not too close" to the yes-instances. $\Sigma$-protocols for these problems were recently proposed in [19], where the generation of hard instances is also addressed. Again, more details are given in [10].

These languages give rise to concrete instantiations of the commitment scheme developed in the above section, based on concrete computational assumptions.

\section{Quantum Zero-Knowledge Proofs}

\subsection{Common-Reference-String Model}

The common-reference-string (CRS) model assumes that there is a string $\sigma$ (honestly) generated according to some distribution and available to all parties from the start of the protocol. In the CRS model, an interactive proof (or argument) is (Q)ZK if there exists a simulator which can simulate the (possibly dishonest) verifier's view of the protocol execution together with a CRS $\sigma$ having correct joint distribution as in a real execution.

\subsection{Efficient QZK Arguments}

We show how to convert any HVZK $\Sigma$-protocol into a quantum zero-knowledge (QZK) argument. The construction is based on a trapdoor commitment scheme and can be proven secure in the CRS model.

It is actually very simple. $\mathrm{P}$ and $\mathrm{V}$ simply execute the $\Sigma$-protocol, but instead of sending message $a$ in the first move, $\mathrm{P}$ sends a commitment to $a$, which he then opens when he sends the answer $z$ to the challenge $c$ in the third move. The zero-knowledge property then follows essentially by observing that the simulator (who knows the trapdoor of the commitment scheme) can cheat in the opening 
of the commitment. So far, the strategy for the QZK proof is the same as in Damgård's concurrent ZK proof [9]; the proof of soundness however will be different since [9] requires to rewind the prover, which cannot be justified in our case by the no-quantum-rewinding paradigm. In order not to rely on the special HVZK property (as introduced and explained in Sect. 3.2), the protocol is slightly more involved than sketched here, though the idea remains.

Let a HVZK $\Sigma$-protocol $\Pi=\left(\right.$ a, c, z) for a language $L=L_{R}$ be given. Let $\epsilon$ denote its soundness error. We assume without loss of generality that a and $c$ sample first messages $a$ and challenges $c$ of fixed bit lengths $r$ and $t$, respectively. Furthermore, let an unconditionally hiding and computationally Q-binding trapdoor commitment scheme $(\mathcal{G}$, commit) be given (where the knowledge of the trapdoor allows to break the binding property of the scheme). We assume that its domain $\mathcal{S}$ contains $\{0,1\}^{r+t}$. Consider Protocol 1 illustrated in Fig. 3.

Protocol 1: $\mathrm{V}$ has input $x$, claimed to be in $L$; $\mathrm{P}$ has input $x$ and $w \in W_{R}(x)$. The CRS is set to be $p k$ where $p k$ is generated by $\mathcal{G}$.

1. P computes $a \leftarrow$ a and chooses $c_{P} \leftarrow \mathrm{c}$. Then it commits to the concatenation $a \| c_{P}$ of $a$ and $c_{P}$ by $C=$ commit $_{p k}\left(a \| c_{P}, \rho\right)$, and sends $C$ to $\mathrm{V}$.

2. $\mathrm{V}$ chooses $c_{V} \leftarrow \mathrm{c}$ and sends it to $\mathrm{P}$.

3. $\mathrm{P}$ computes $z \leftarrow \mathbf{z}_{x}(a, c)$ for $c=c_{P} \oplus c_{V}$ and sends $\left(a, c_{P}, \rho\right)$ and $z$ to $\mathrm{V}$.

4. $\mathrm{V}$ accepts iff $C=\operatorname{commit}\left(a \| c_{P}, \rho\right)$ and verify ${ }_{x}\left(a, c_{P} \oplus c_{V}, z\right)=$ accept.

Fig. 3. QZK proof protocol in the CRS model.

As mentioned above, Protocol 1 can be slightly simplified in case $\Pi$ is special HVZK in that $\mathrm{P}$ commits to $a$ (rather than to $a \| c_{P}$ ) and computes $z$ with respect to the challenge $c=c_{V}$ provided by $\mathrm{V}$.

Theorem 2. Under the assumption that (G, commit) is an unconditionally hiding and computationally Q-binding trapdoor commitment scheme, Protocol 2 is a QZK (quantum) argument for $L$ in the CRS model. Its soundness error is $\epsilon^{\prime}=\epsilon+$ negl where negl is negligible (in the security parameter).

Concerning the flavor of QZK, Protocol 2 is computational QZK if the underlying $\Sigma$-protocol $\Pi$ is computational HVZK, and it is statistical QZK provided that $\Pi$ is statistical or perfect HVZK. In case $(\mathcal{G}$, commit) is perfectly (rather than unconditionally) hiding, the flavor of QZK of Protocol 2 is exactly given by the flavor of HVZK of $\Pi$.

Proof. As mentioned above, the zero-knowledge property is rather straight forward: The simulator generates a public-key for the commitment scheme together with a trapdoor and outputs the public-key as CRS. Then, on input $x \in L$, it generates a commitment $C$ (which he can open to an arbitrary value using the trapdoor) and sends it to $\tilde{\mathrm{V}}$. On receiving $c_{V}$ from $\tilde{\mathrm{V}}$, the simulator simulates 
an accepting conversation $(a, c, z)$ for the original $\Sigma$-protocol using the HVZK property, it sets $c_{P}=c \oplus c_{V}$ and computes $\rho$ such that $C=\operatorname{commit}\left(a \| c_{P}, \rho\right)$ using the trapdoor, and it sends $\left(a, c_{P}, \rho\right)$ and $z$ to $\tilde{\mathrm{V}}$.

For the soundness property, it has to be shown that given a (quantum) prover $\tilde{\mathrm{P}}$, which succeeds in making (honest) $\mathrm{V}$ accept the proof for an $x \notin L$ with a probability exceeding $\epsilon$ by a non-negligible amount, $\tilde{\mathrm{P}}$ can be used to break the Q-binding property of the commitment scheme for some predicate $Q$. Fix $x \notin L$. We define $Q$ as follows. $N=1$, and $\mathcal{U}$ is given by the set of all possible challenges $c_{V}$ sampled by c. For $s \in \mathcal{S}$ and $u=c_{V} \in \mathcal{U}$, where $s$ is parsed as $s=a \| c_{P}$ with $a \in\{0,1\}^{r}$ and $c_{P} \in\{0,1\}^{t}$, we set $Q(\{1\}, s, u)=1$ if and only if the challenge $c=c_{P} \oplus c_{V}$ for the first message $a$ allows an answer $z$ such that verify $_{x}(a, c, z)=$ accept. Note that $A=\{1\}$ is the only legitimate choice for $A$. By construction of $Q$, making $\mathrm{V}$ accept the proof means that $\tilde{\mathrm{P}}$ opens $C$ (correctly) to $a \| c_{P}$ such that $Q\left(\{1\}, a \| c_{P}, c_{V}\right)=1$. Furthermore, $p_{\text {IDEAL }}=\epsilon$. It follows that if $\tilde{P}$ succeeds in making $V$ accept the proof with probability greater that $\epsilon$ by a non-negligible amount, then $\tilde{\mathrm{P}}$ is a forger $\mathcal{F}$ that breaks the Q-binding property of (G,commit). This completes the proof.

\subsection{QZK Arguments for All of NP}

Consider a (generic) ZK argument for an NP-complete language using (ordinary) unconditionally hiding commitments. For instance, consider the classical interactive proof for Circuit-Satisfiability due to Brassard, Chaum and Crépeau [3]: the prover "scrambles" the wires and the gates' truth tables of the circuit and commits upon it, and he answers the challenge $c=0$ by opening all commitments and showing that the scrambling is done correctly and the challenge $c=1$ by opening the (scrambled) wires and rows of the gates' truth tables that are activated by the satisfying input. Following the lines of the proof of Theorem 2 above, it is straightforward to prove that replacing the commitment scheme in this construction by an unconditionally hiding and computationally Q-binding commitment scheme results in a QZK argument in the CRS model for CircuitSatisfiability, and thus for all languages in $N P$.

\subsection{Computational QZK Proofs}

We sketch how to construct rather efficient computational QZK proofs for languages that allow (computational) HVZK $\Sigma$-protocols based on specific intractability assumptions, as well as computational QZK proofs for all of $N P$ based on any quantum one-way function.

Consider any of the languages $L=L_{R}$ with HVZK $\Sigma$-protocol on which the commitment construction from Sect. 3.2 is based, except that we allow the $\Sigma$-protocol to be computational HVZK. Assume in addition that there is also a generator $\mathcal{G}_{\text {no }}$ that produces no-instances that cannot be distinguished from the yes-instances produced by $\mathcal{G}_{\text {yes }}$.

Then, put a no-instance $x_{\text {no }}$ in the reference string. The prover can now prove any statement $S$ that can be proved by an HVZK $\Sigma$-protocol $\Pi$ by us- 
ing a standard witness-indistinguishable HVZK proof for proving that $S$ is true or $x_{\text {no }} \in L$ [7]. Here, we allow the $\Sigma$-protocol $\Pi$ to be computational HVZK, in particular $\Pi$ might be the $\Sigma$-protocol for Circuit-Satisfiability sketched in Sect. 4.3 above but based on an unconditionally binding and computationally hiding commitment scheme (secure against quantum attacks), which can be constructed from any (quantum) one-way function (see below).

This is clearly unconditionally sound, and can be simulated, where the simulator uses a yes-instance $x_{\text {yes }}$ in place of $x_{\text {no }}$ and uses its witness $w \in W_{R}\left(x_{\text {yes }}\right)$ to complete the protocol without rewinding. A distinguisher would have to contradict the HVZK property of one of the underlying $\Sigma$-protocols, or the indistinguishability of yes- and no-instances.

This can be instantiated efficiently if we are willing to assume about the coding or lattice problem or some other candidate problem that it also satisfies this stronger version of indistinguishability of yes- and no-instances. But it can also be instantiated in a version that can be be based on any one-way function: First, the (unconditionally binding and computationally hiding) commitment scheme of Naor [20] is also secure against quantum adversaries, and exists if any one-way function exists. So consider the language of pairs $(p k, O)$ where $p k$ is a public-key for the commitment scheme and $O$ is a commitment of 0 . This language has a computational HVZK $\Sigma$-protocol using generic ZK techniques, driven by Naor's commitments. Furthermore, the set of no-instances $(p k, E)$ where $E$ is a commitment to 1 is easy to generate and hard to distinguish from the yes-instances.

\section{Relaxed Honest-Verifier Quantum Proofs}

It is a natural question whether QZK proof systems exist without having to rely upon common reference strings. In this section, we answer this question partially. We define a quantum interactive proof system associated to any $\Sigma$ protocol. Our scheme is QZK against a relaxed version of honest verifiers that we call non-oblivious. Intuitively, a non-oblivious verifier is a verifier having access to the same classical variables than the honest verifier. We show that any HVZK $\Sigma$-protocol can be turned into a non-oblivious verifier QZK proof using quantum communication.

\subsection{Quantum Circuits for $\Sigma$-Protocols}

Assume $L=L_{R}$ has a classical HVZK $\Sigma$-protocol $\Pi=($ a, c, z). We specify unitary transforms $Z_{x}(a)$, and $T_{x}(a)$, depending on $a \leftarrow$ a, which implement quantum versions of the computations specified by z and verify. Throughout, we assume without loss of generality that $c$ samples $c$ uniformly from $\{0,1\}^{t}$ for some $t$.

The answer $z \leftarrow \mathrm{z}_{x}(a, c)$ to challenge $c$ when $a$ was announced during the first round can be computed quantumly through some unitary transform $Z_{x}(a)$ depending upon the initial announcement $a$. That is, provided quantum registers $P$ and $X$, we have:

$$
Z_{x}(a):|c\rangle^{P}|y\rangle^{X} \mapsto|c\rangle^{P}\left|y \oplus \mathbf{z}_{x}(a, c)\right\rangle^{X} .
$$


Similarly, the testing process performed by $\mathrm{V}$ can also be executed by a quantum circuit $T_{x}(a)$ depending on the announcement of $a$. Transformation $T_{x}(a)$ stores the output of the verification process in an extra one-qubit register $T$ :

$$
T_{x}(a):|z\rangle^{X}|c\rangle^{V}|t\rangle^{T} \mapsto|z\rangle^{X}|c\rangle^{V}\left|t \oplus \operatorname{verify}_{x}(a, c, z)\right\rangle^{T} .
$$

If $z \leftarrow \mathrm{z}_{x}(a, c)$ and verify ${ }_{x}(a, c, z)$ can be classically computed in polynomial time (given the randomness of the computation of $a$ and a witness $w \in W_{R}(x)$ for the former), circuits $Z_{x}(a)$ and $T_{x}(a)$ can be implemented by poly-size quantum circuits.

\subsection{EPR-Pairs Based Proofs}

The idea behind the protocol is as follows. P chooses $a \leftarrow$ a and sends the answer to all possible challenges in quantum superposition to $\mathrm{V}$. $\mathrm{V}$ then verifies quantumly that all answers in the superposition are correct. In a further step, $\mathrm{P}$ convinces $\mathrm{V}$ that the state contains the answer to more than one challenge. Since $\Pi$ is assumed to be special sound, it follows that $x \in L$.

Concretely, $P$ starts by choosing $a \leftarrow$ a and by preparing $t$ EPR pairs in state:

$$
\left|\Omega_{t}\right\rangle^{P, V}=2^{-t / 2} \sum_{c \in\{0,1\}^{t}}|c\rangle^{P}|c\rangle^{V}=2^{-t / 2} \sum_{c \in\{0,1\}^{t}}|c\rangle_{\times}^{P}|c\rangle_{\times}^{V} .
$$

The two equivalent ways of writing $\left|\Omega_{t}\right\rangle$ shows that it exhibits the same correlation between registers $P$ and $V$ in both the computational and the diagonal bases. This property will be used later in the protocol. Now, P adds an extra register $X$ initially in state $|0\rangle^{X}$ before applying $Z_{x}(a)$ upon registers $P$ and $X$. This results in state,

$$
\left|\psi_{a}\right\rangle=2^{-t / 2} \sum_{c \in\{0,1\}^{t}} Z_{x}(a)|c\rangle^{P}|0\rangle^{X} \otimes|c\rangle^{V}=2^{-t / 2} \sum_{c \in\{0,1\}^{t}}|c\rangle^{P}|z\rangle^{X} \otimes|c\rangle^{V}
$$

where every $z$ in the superposition is computed as $z \leftarrow z_{x}(a, c)$. P then announces $a$ and sends registers $V$ and $X$ to $\mathrm{V}$ allowing him to apply the verification quantum circuit $T_{x}(a)$ after adding an extra register $T$ initially in state $|0\rangle^{T}$. That is,

$$
\begin{aligned}
\left|\psi_{a}^{T}\right\rangle & =\left(\mathbb{I}_{P} \otimes T_{x}(a)\right)\left|\psi_{a}\right\rangle|0\rangle^{T}=2^{-t / 2} \sum_{c \in\{0,1\}^{t}}|c\rangle^{P} \otimes T_{x}(a)|z\rangle^{X}|c\rangle^{V}|0\rangle^{T} \\
& \left.=2^{-t / 2} \sum_{c \in\{0,1\}^{t}}|c\rangle^{P} \otimes|z\rangle^{X}|c\rangle^{V} \mid \text { verify }_{x}(a, c, z)\right\rangle^{T}=\left|\psi_{a}\right\rangle \otimes|0\rangle^{T}
\end{aligned}
$$

$\mathrm{V}$ then measures register $T$ in the computational basis and rejects if $|0\rangle^{T}$ is not observed. Provided $P$ was honest, the test will always be successful by assumption on the original $\Sigma$-protocol $\Pi$, and the verification process does not affect the state $\left|\psi_{a}\right\rangle$. V then returns register $X$ back to $\mathrm{P}$, who can recover $t$ shared EPR pairs by running $Z_{x}(a)^{\dagger}$, the inverse of $Z_{x}(a)$. Finally, $\mathrm{P}$ measures register $P$ in 
the diagonal basis and announces the outcome to $\mathrm{V}$. $\mathrm{V}$ does the same to register $V$ and verifies that the same outcome is obtained. By the properties of EPR pairs (1), it follows that the measurements coincide provided $\mathrm{P}$ was honest. A compact description of the protocol is given by Protocol 2 in Fig. 4 .

Protocol 2: $\mathrm{V}$ has input $x$, claimed to be in $L$; P has input $x$ and $w \in W_{R}(x)$.

1. P computes a first message $a \leftarrow$ a and prepares the quantum state $\left|\psi_{a}\right\rangle^{P, X, V}=2^{-t / 2} \sum_{c}|c\rangle^{P}|z\rangle^{X}|c\rangle^{V}$ as in (2) where $z \leftarrow \mathbf{z}_{x}(a, c)$, and he sends $a$ and the registers $X$ and $V$ to $\mathrm{V}$,

2. $\mathrm{V}$ runs the verification circuit $T_{x}(a)$ and rejects if a non-zero outcome is obtained. If the test was successful then $\mathrm{V}$ returns register $X$ to $\mathrm{P}$,

3. P runs $\left(Z_{x}(a)^{\dagger} \otimes \mathbb{I}_{V}\right)\left|\psi_{a}\right\rangle=\left|\Omega_{t}\right\rangle^{P, V} \otimes|0\rangle^{X}$, measures the $P$ register in the diagonal basis and announces the outcome $c_{P} \in\{0,1\}^{t}$ to $\mathrm{V}$.

4. $\mathrm{V}$ accepts iff register $V$ measured in the diagonal basis produces outcome $c_{V}=c_{P}$.

Fig. 4. Non-oblivious verifier QZK proof.

\subsection{Soundness}

Consider $x \notin L$. We show that in Protocol 2, any prover $\tilde{\mathrm{P}}$ has probability at most $2^{-t}$ to convince $\mathrm{V}$, given that $\Pi$ is special sound. Let $a$ be announced by $\tilde{P}$ at step 1 . By the special soundness property of $\Pi$, if $\tilde{P}$ passes the test at step 2. then the state shared between $\tilde{P}$ and $V$ is of the following form: $\left|\tilde{\psi}_{a}\right\rangle=\left|\gamma_{a, x}\right\rangle^{P, X} \otimes|c\rangle^{V}|0\rangle^{T}$, where $c$ is the unique challenge that can be answered given the announcement of $a$. Since after register $X$ has been sent back to $\tilde{\mathrm{P}}$, register $V$ is in pure state, it follows that only one answer is possible when $V$ is measured in the computational basis. That is, $|c\rangle$ is guaranteed to be observed. However, V's final test involves a measurement of that same register in the diagonal basis, and it is easy to see that the outcome of a measurement in the diagonal basis applied to $|c\rangle$ is uniformly distributed over $\{0,1\}^{t}$. This is a special case of the entropic uncertainty relations [18]. It follows:

Theorem 3. If $\Pi$ is a special-sound $H V Z K \Sigma$-protocol for language $L=L_{R}$ where c samples in $\{0,1\}^{t}$, then Protocol 2 is a quantum interactive proof for $L$ with soundness error $2^{-t}$.

It should be mentioned that $\Pi$ being special sound is not a strict necessary condition for Protocol 2 to be sound. A more careful analysis can handle the case where $\Pi$ is "not too far away" from special sound. For simplicity, in this paper we only address the case of special sound $\Sigma$-protocols.

\subsection{Non-oblivious Verifier Quantum Zero-Knowledge}

Classical $\Sigma$-protocols with large challenges are not known to be ZK against a dishonest verifier. This is due to the fact that rewinding allows the simulator 
to succeed only if it has a non-negligible probability to guess the challenge that the verifier will pick. This is true even with respect to verifiers that submit a uniformly distributed challenge $c \in\{0,1\}^{t}$ and are able to do the verification test as prescribed. To see this, let $\sigma:\{0,1\}^{\ell} \rightarrow\{0,1\}^{\ell}$ be a one-way permutation and let us assume for simplicity that $t=\ell$ and a samples $a$ from $\{0,1\}^{t}$. If $\tilde{V}$ announces challenge $c=a \oplus \sigma(m)$ for random $m \in\{0,1\}^{\ell}$ and $a \leftarrow$ a announced by $\mathrm{P}$ as first message, then the simulator must generate $(a, c, z, m)$ since it is part of $\tilde{V}$ 's view. However, the simulator typically can compute a only after having picked $c$, which means that it has to compute $m$ as $m=\sigma^{-1}(c \oplus a)$. Note that even though $c \oplus a$ is not necessarily uniformly distributed, it seems that the simulator has typically not enough control over the value $c \oplus a$ in order to compute $m$.

Notice that a verifier $\tilde{V}$ acting as described above rejects a false statement with the same probability and chooses the challenge $c$ with the same distribution as an honest verifier, yet there is no known efficient simulator for $\tilde{V}$. In this section we show that Protocol 2 is quantum zero-knowledge provided that $\tilde{V}$ is non-oblivious of the value $c_{V}$ needed for the verification at step 4 . More generally, we define non-oblivious verifiers the following way:

Definition 2. A verifier $\tilde{\mathrm{V}}$ is said to be non-oblivious if it produces the same (public and private) variables as honest $\mathrm{V}$ according the same distribution.

As illustrated above, in contrast to an honest verifier a non-oblivious verifier can produce his variables in an arbitrary manner, as long as they are correctly distributed.

In Protocol 2, a non-oblivious verifier $\tilde{V}$ has access to the string $c_{V}$ so it can be made available to the simulator. Indeed, this allows to produce a simulation of the interaction between $P$ and $\tilde{V}$. It is straightforward to verify that the simulator described in Fig. 5 generates the same view as when $\tilde{V}$ interacts with P:

Simulator: Input is $x \in L$.

1. Run the HVZK simulator for $\Pi$ in order to get triplet $(a, c, z)$, and send $a$ together with the quantum state $|c\rangle|z\rangle$ to $\tilde{\mathrm{V}}$,

2. If $\tilde{V}$ rejects $P$ then halt, otherwise throw away the state sent by $\tilde{V}$,

3. Extract $c_{V}$ using the non-obliviousness of $\tilde{\mathrm{V}}$ and announce $c_{P}=c_{V}$.

Fig. 5. Simulator for Protocol 2.

Theorem 4. Protocol 2 built from a special-sound (statistical/perfect) HVZK $\Sigma$-protocol $\Pi$ is (statistical/perfect) $Q Z K$ provided $\tilde{\mathrm{V}}$ is non-oblivious.

A weaker assumption about $\tilde{V}$ 's behavior would be obtained if the only constraint was that $\tilde{V}$ detects false statements with the same probability as the honest verifier $\mathrm{V}$. Let us say that such a verifier is verification-enabled. In general, a verification-enabled verifier $\tilde{V}$ is not necessarily non-oblivious since in 
order to verify $\tilde{P}$ 's announcement, $c_{P}$ does not necessarily have to be determined by $\tilde{V}$ without P's help. However, it can be shown that for $\Sigma$-protocols with challenges of polylogarithmic size, any verification-enabled $\tilde{V}$ in Protocol 2 is also non-oblivious.

\section{Acknowledgements}

The authors are grateful to Claude Crépeau for having introduced the problem to one of us and discussed its relevance. We would also like to thank Jesper Nielsen for enlightening discussions.

\section{References}

1. Barak, B., How to Go Beyond the Black-box Simulation Barrier, in 42th Annual Symposium on Foundations of Computer Science (FOCS), 2001.

2. Brassard, G., and C. Crépeau, Zero-Knowledge Simulation for Boolean Circuits, in Advances in Cryptology - CRYPTO 86, Lecture Notes in Computer Science, vol. 263, Springer-Verlag, 1987.

3. Brassard, G., D. Chaum, and C. Crépeau, Minimum Disclosure Proofs of Knowledge, JCSS, 37(2), 1988.

4. Blum, M., P. Feldman and S. Micali, Non-Interactive Zero-Knowledge and Its Applications, in 20th Annual Symposium on Theory Of Computing (STOC), 1988.

5. CAnetti, R., Universally Composable Security: A New Paradigm for Cryptographic Protocols, in 42th Annual Symposium on Foundations of Computer Science (FOCS), 2001.

6. Canetti, R., and M. Fischlin, Universally Composable Commitments, in Advances in Cryptology - CRYPTO 01, Lecture Notes in Computer Science, vol. 2139, Springer-Verlag, 2001.

7. Cramer, R., I. Damgård, and B. Schoenmakers, Proofs of Partial Knowledge and Simplified Design of Witness Hiding Protocols, in Advances in Cryptology CRYPTO 94, Lecture Notes in Computer Science, vol. 839, Springer-Verlag, 1994.

8. Crépeau, C., P. Dumais D. Mayers and L. Salvail, Computational Collapse of Quantum State with Application to Oblivious Transfer, in Advances in Cryptology - TCC 04, Lecture Notes in Computer Science, vol. 2951, Springer-Verlag, 2004.

9. DAMGÅRD, I., Efficient Concurrent Zero-Knowledge in the Auxiliary String Model, in Advances in Cryptology - EUROCRYPT 00, Lecture Notes in Computer Science, vol. 1807, Springer-Verlag, 2000.

10. DamgÅRD, I.,S. Fehr, and L. SAlvail,Zero-Knowledge Proofs and String Commitments Withstanding Quantum Attacks, full version of this paper, BRICS report nr. RS-04-9, available at www.brics.dk/RS/04/9, 2004.

11. Damgånd, I., and J. Nielsen, Perfect Hiding and Perfect Binding Universally Composable Commitment Schemes with Constant Expansion Factor, in Advances in Cryptology - CRYPTO 02, Lecture Notes in Computer Science, vol. 2442, Springer-Verlag, 2002.

12. Dumais, P., D. Mayers, and L. Salvail, Perfectly Concealing Quantum Bit Commitment From Any Quantum One-Way Permutation, in Advances in Cryptology - EUROCRYPT 00, Lecture Notes in Computer Science, vol. 1807, Springer-Verlag, 2000. 
13. Dwork, C., M. NAor, and A. SAhai, Concurrent Zero-Knowledge, in 30th Annual Symposium on Theory Of Computing (STOC), 1998.

14. Goldwasser, S., S. Micali, and C. Rackoff, The Knowledge Complexity of Interactive Proof Systems, in 17th Annual Symposium on Theory Of Computing (STOC), 1985.

15. Goldreich, O., S. Micali, and A. Wigderson, Proofs that Yield Nothing but their Validity, or All Languages in NP Have Zero-Knowledge Proof Systems, J. ACM., 38(3), 1991.

16. Fiat, A., and A. Shamir, How to Prove Yourself: Practical Solutions to the Identification and Signature Problem, in Advances in Cryptology - CRYPTO 86, Lecture Notes in Computer Science, vol. 263, Springer-Verlag, 1987.

17. Kitaev, A., and J. Watrous, Parallelization, Amplification, and Exponential Time Simulation of Quantum Interactive Proof Systems, in 32nd Annual Symposium on Theory of Computing (STOC), 2000.

18. Matssen, H., and J.B.M. Uffink, Generalized Entropic Uncertainty Relations, Phys. Rev. Letters, vol. 60, 1988.

19. Micciancio, D., and S. P. Vadhan, Statistical Zero-Knowledge Proofs with Efficient Provers: Lattice Problems and More, in Advances in Cryptology CRYPTO 03, Lecture Notes in Computer Science, vol. 2729, Springer-Verlag, 2003.

20. NAor, M., Bit Commitment Using Pseudorandomness, Journal of Cryptology, vol. 4, no. 2, 1991.

21. Pfitzmann, B., and M. Waidner, Composition and Integrity Preservation of Secure Reactive Systems, in 7th ACM Conference on Computer and Communications Security, 2000.

22. Richardson, R. and J. Kilian, On the Concurrent Composition of ZeroKnowledge Proofs, in Advances in Cryptology - EUROCRYPT 99, Lecture Notes in Computer Science, vol. 1592, Springer-Verlag, 1999.

23. Shor, P., Algorithms for Quantum Computation: Discrete Logarithms and Factoring, in 35th Annual Symposium on Foundations of Computer Science (FOCS), 1994.

24. Watrous, J,PSPACE has Constant-Round Quantum Interactive Proof Systems, in 40th Annual Symposium on Foundations of Computer Science (FOCS), 1999.

25. Watrous, J.,Succinct Quantum Proofs for Properties of Finite Groups, Proceedings of the 41st Annual Symposium on Foundations of Computer Science, 2000.

26. Watrous, J., Limits on the Power of Quantum Statistical Zero-Knowledge, in 43rd Annual Symposium on the Foundations of Computer Science (FOCS), 2002.

27. van de GraAf, J., Towards a Formal Definition of Security for Quantum Protocols, Ph.D. thesis, Computer Science and Operational Research Department, Université de Montréal, 1997. 